\title{
MODELAGEM COMPUTACIONAL SEMIQUANTITATIVA DE SISTEMAS FÍSICOS: UMA EXPERIÊNCIA NO ENSINO MÉDIO
}

\author{
Valter Antonio Ferreira, UNIPAMPA, valterferreira@unipampa.edu.br \\ Lisete Funari Dias, UNIPAMPA, lisetedias@unipampa.edu.br \\ Vera Lúcia Duarte Ferreira, UNIPAMPA, veraferreira@unipampa.edu.br
}

\begin{abstract}
Resumo: Este estudo sobre aprendizagem colaborativa apoiada por computador, investigou a utilização do Ambiente de Modelagem Computacional Semiquantitativa Baseado na Metáfora de Ícones (WlinkIt), articulando alguns sistemas físicos com atividades de modelagem e simulação, envolvendo alunos do Ensino Médio do Colégio Municipal Pelotense, no município de Pelotas, RS. A investigação ocorreu por meio de um curso de extensão sobre modelagem de sistemas físicos, no qual os alunos atuaram em 05 duplas. Os instrumentos de coleta de dados foram a observação dos diálogos e dos comportamentos dos estudantes e a análise documental do material textual produzido pelos mesmos. Os resultados sugerem que os estudantes apresentaram a capacidade de utilizar e desenvolver o raciocínio semiquantitativo, além de atitudes de cooperação e autonomia.
\end{abstract}

Palavras-chave: modelagem computacional, ensino de física, informática educativa.

\begin{abstract}
This study about collaborative learning supported by computer investigated the use of the Semiquantitative Computational Modeling Environment Based on the Iconic Metaphor (WlinkIt). The study design articulated some physical systems with modeling and simulation activities. The study was implemented at Colégio Municipal Pelotense with high-school students. To carry out the study, a course was designed. The students worked in 5 pairs. As data collection instruments, first of all, observations of dialogs and behavior of students and documental analyses of the textual material produced by the students. The results suggest that the students were able to develop and use the semiquantitative reasoning, besides behaving in a cooperative and autonomous way.
\end{abstract}

Keywords: computational modeling, physics education, informatics education.

\section{INTRODUÇÃO}

No contexto do Ensino Médio, a aprendizagem de Física pode ser considerada como um dos pilares para o desenvolvimento intelectual dos futuros cidadãos de um país. Via de regra, os processos de ensino e de aprendizagem de Física ainda abordam os fenômenos apenas pelo viés matemático, culminando somente em uma solução analítica. Nessa perspectiva, alguns estudantes, por apresentarem deficiências quanto ao domínio do formalismo matemático, dificilmente compreendem completamente as relações existentes em determinado sistema físico em estudo, (Camiletti, 2001).

No entanto, diferentes métodos e estratégias educacionais têm sido propostos na busca pela qualificação da aprendizagem de Física no Nível Básico. Um destes métodos propõe abordar os fenômenos físicos dispensando, inicialmente, um conhecimento matemático mais aprofundado, através de atividades envolvendo o chamado Raciocínio Semiquantitativo ${ }^{1}$. Entre as possíveis vantagens dessa proposta, está a possibilidade de

\footnotetext{
${ }^{1}$ Raciocínio semiquantitativo é o estabelecimento de relações de influência entre as variáveis de um sistema, sem a necessidade de calcular valores numéricos. Por exemplo, o aumento da quantidade de vapor interno de uma panela de pressão, causará um aumento na pressão interna da mesma e, como
} 
sua aplicação desde as séries iniciais até o nível universitário, visto que não exige do aluno um profundo conhecimento matemático para a compreensão do sistema ${ }^{2}$. Além disso, estudos realizados por Kurtz dos Santos (1995) apontam para o raciocínio semiquantitativo como um estágio de pré-formalismo matemático, resultando em uma melhor preparação do estudante para uma posterior abordagem quantitativa dos sistemas físicos.

Dessa forma, esse tipo de raciocínio pode ser desenvolvido através de atividades de modelagem, ou seja, relacionar entidades importantes de um sistema como variáveis, eventos ou objetos, objetivando a construção de um modelo ${ }^{3}$, o qual poderá auxiliar o aluno a organizar, compreender e estruturar seu conhecimento sobre o tema em estudo (Ogborn, 1992).

A partir desse contexto, a tecnologia da informática busca introduzir uma forma de estudo de sistemas físicos utilizando soluções computacionais, pois a animação de variáveis e as saídas gráficas podem auxiliar na compreensão de sistemas mais complexos, onde muitas variáveis atuam ao mesmo tempo entre si (Sampaio,1999). A adequação do software aponta para um ambiente de modelagem computacional baseado na metáfora icônica, ou seja, as variáveis envolvidas no estudo e as possíveis ligações entre elas são representadas por ícones, sendo os cálculos necessários para animar o modelo realizados por procedimentos computacionais internos ao software, liberando o estudante da carga cognitiva exigida pela programação e pelo conhecimento matemático (Machado, 1999). Em outras palavras, é construído um modelo icônico o qual é traduzido em informação simbólica, a partir da qual são executados cálculos e feitas inferências cujos resultados são novamente traduzidos para a forma icônica.

Neste sentido, este artigo aborda um estudo sobre o uso da modelagem computacional semiquantitativa no estudo de sistemas físicos, realizada com estudantes do Nível Médio do Colégio Municipal Pelotense, no município de Pelotas-RS. Ao longo da investigação procurou-se encontrar respostas para algumas indagações, entre elas a possibilidade da modelagem computacional estimular desenvolvimento do Raciocínio Semiquantitativo em estudantes do Ensino Médio; a influência da aprendizagem colaborativa na qualidade do processo de interação dos alunos entre si, com o software e com o professor e as limitações do software WlinkIt, durante as atividades de modelagem.

\section{MATERIAIS E MÉTODOS}

Nesta pesquisa trabalhou-se com dez alunos, divididos em cinco duplas. Não houve escolha dos sujeitos, pois foram abertas inscrições para um curso de extensão denominado "Modelagem computacional semiquantitativa de sistemas físicos", realizado em turno inverso ao dos estudantes. Dessa forma, apresentaram-se as seguintes duplas: três do primeiro ano noturno (duplas 01, 02 e 03), uma do segundo ano noturno (dupla 04) e uma do terceiro ano diurno (dupla 05). Tais duplas trabalharam separadamente em quatro encontros, de duas horas cada, por dupla, totalizando 40 horas de curso para as 05 duplas. Os alunos envolveram-se com um total

consequência, a atuação da válvula de escape de vapor, diminuindo a pressão interna da panela (Kurtz dos Santos, 1994).

${ }^{2}$ Sistema é um agrupamento de partes que operam juntas com um propósito comum e pode representar qualquer realidade conhecida, desde o átomo até a galáxia, passando pela molécula, pela célula e pelo organismo, juntamente com a própria sociedade (Morin, 1991).

3 Modelo tende a ser uma representação simplificada de um sistema, auxiliando no entendimento do mesmo, porém não sendo uma cópia fiel da realidade observada, mas uma edificação fruto de um sistema conceitual e de uma interpretação individual (Machado, 1999). 
de 22 modelos (03 exploratórios e 19 expressivos). Através de alguns questionamentos iniciais, foi possível verificar que somente os alunos das duplas 01 e 05 possuíam computador em casa, as demais tinham apenas uma pequena experiência com Windows e Word, através de aulas de algumas disciplinas que utilizavam, esporadicamente, o laboratório de Informática da escola. As idades variaram entre 16 e 19 anos e todos os estudantes pertenciam às camadas populares da população.

A escolha por realizar o trabalho com duplas foi feita com base nos estudos de Kurtz dos Santos (1995), pois com relação à pesquisa em Educação com modelagem computacional, esse autor privilegia o trabalho aos pares, por considerá-lo benéfico à aprendizagem, já que a interação social entre a dupla pode constituir-se em fator facilitador da compreensão e da execução das atividades exploratórias ${ }^{4}$ e expressivas ${ }^{5} \cdot$. O mesmo autor observa, a partir de sua própria experiência, que o estudante sozinho no computador, pode sentir-se intimidado tanto pelo uso do software quanto pela presença do pesquisador. Tal situação pode tornar-se prejudicial, principalmente nas atividades expressivas, pela impossibilidade da troca de idéias entre companheiros, dificultando a evolução do modelo criado. Por outro lado, quando uma dupla pode trocar opiniões sobre certo sistema, nota-se uma maior fluidez no trabalho e o desenvolvimento do respectivo modelo evolui com maior facilidade.

O curso desenvolveu-se em três módulos, respectivamente, Ensino de modelagem, Atividades de Modelagem Exploratória e Atividades de Modelagem Expressiva. Na fase de ensino, foram expostas aos alunos algumas definições e exemplos sobre sistemas, modelos e modelagem, objetivando estimular a reflexão acerca dos entendimentos dos alunos sobre o conceito de "modelo", visto que, grande parte dos estudantes não concebe o modelo computacional como uma das várias maneiras de ver e interpretar "a realidade", mas tratam-no como o próprio fenômeno estudado (Kurtz dos Santos, 1995).

$\mathrm{Na}$ seqüência, os estudantes tiveram contato com a teoria básica sobre os diagramas de influência ${ }^{6}$, com e sem elo de retroalimentação ${ }^{7}$. Depois, executaram 16 exercícios sobre a construção de tais diagramas, durante os quais foram sanadas dúvidas e explicadas, a função, os efeitos e a identificação de um elo de retroalimentação, negativo ou positivo. Após, ocorreu a iniciação aos comandos básicos para o manuseio do software WlinkIt ${ }^{8}$. Na fase de modelagem exploratória, os alunos analisaram, descreveram, passaram para a metáfora computacional e validaram modelos (forno de microondas, ar condicionado e submarino) construídos pelo pesquisador. Por sua vez, durante a etapa de modelagem expressiva, os alunos, com base em informações de apoio e em seus conhecimentos prévios, construíram seus próprios modelos (panela de pressão e efeito estufa), os quais foram analisados, descritos, transpostos para a metáfora computacional e validados. Na sequência é apresentado um exemplo de

\footnotetext{
${ }^{4}$ Atividade na qual a dupla analisa um modelo previamente elaborado (Kurtz dos Santos, 1995).

${ }^{5}$ Atividade na qual a dupla constrói o próprio modelo (Kurtz dos Santos, 1995).

${ }^{6}$ Técnica de construção de modelos utilizando palavras e setas para representar do comportamento de um sistema (Mandinach, 1989).

${ }^{7}$ São diagramas de influência com elos fechados, cuja característica pode ser negativa - quando o sistema representado resiste a mudanças, tendendo para a estabilidade - ou positiva - cujos elos auto-reforçantes produzem um crescimento excessivo, chegando ao colapso (Kurtz dos Santos, 1995).

${ }^{8} \mathrm{O}$ software WlinkIt é um ambiente de modelagem computacional para a construção e simulação de modelos dinâmicos semiquantitativos, com base na metáfora icônica. Através desse software, é possível construir modelos representativos das relações de influência entre variáveis importantes de fenômenos, eventos ou objetos do mundo a ser modelado (http://www.modelab.ufes.br/wlinkit) (Sampaio, 1999).
} 
material de apoio, constituído por um texto básico e pela figura 1, como exemplo da atividade expressiva relativa ao efeito estufa realizada pela dupla número 05 .

O efeito estufa

A cobertura de nuvens da Terra reflete cerca de um quarto da radiação proveniente do Sol, devolvendo-a ao espaço, de maneira que esta radiação não participa do aquecimento do nosso planeta. A maior parte da insolação que não é refletida e perdida para o espaço é usada no aquecimento das áreas sólidas e líquidas da Terra. $O$ ritmo acelerado de industrialização e poluição aumentou enormemente a quantidade dos gases responsáveis pelo efeito estufa e afetou o equilíbrio ecológico. Se a quantidade desses gases aumentar muito, a radiação infravermelha retida pela atmosfera crescerá, aumentando a temperatura do planeta em um aquecimento de grandes proporções.

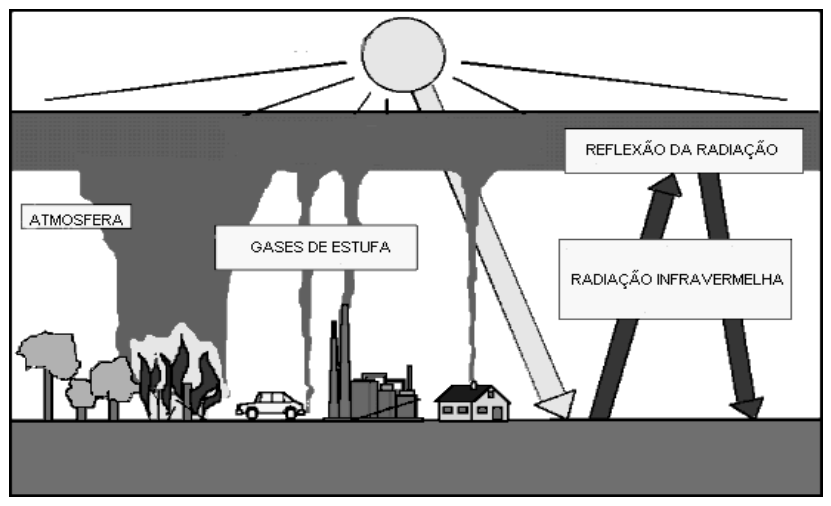

Figura 1- Esquema de aquecimento da Terra.

Após a leitura do texto, a dupla trabalhou para construir um modelo do efeito estufa utilizando diagramas de influência, como pode ser visto na figura 2 .

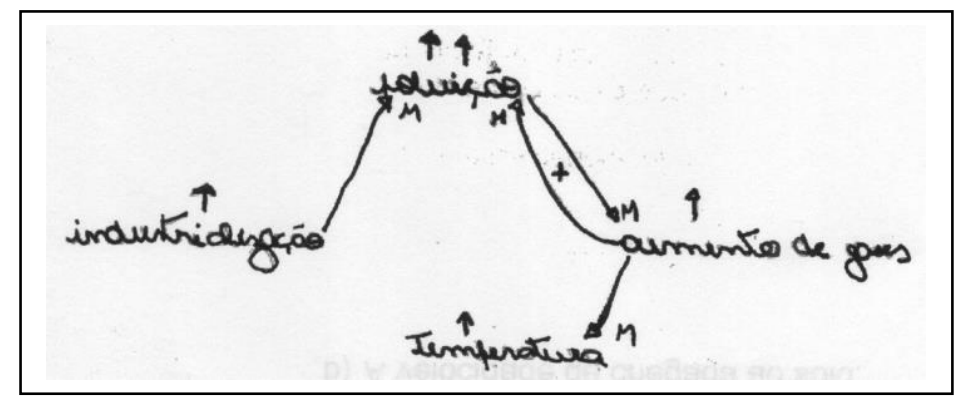

Figura 2 - Diagrama de influências do sistema efeito estufa elaborado pela dupla 05.

A seguir, a figura 3 apresenta um texto escrito pelos alunos sobre o possível funcionamento do modelo criado na figura 2.

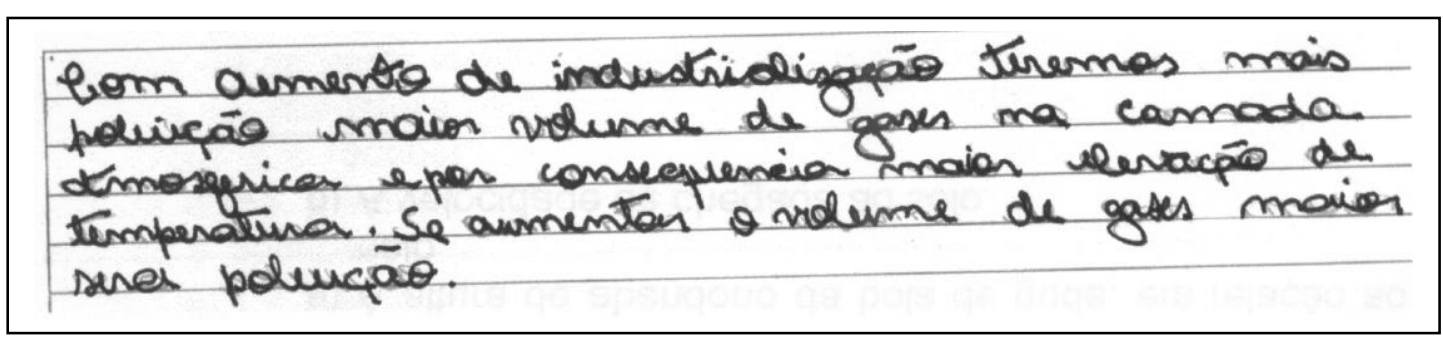

Figura 3 - Texto elaborado pela dupla 05 sobre o comportamento do diagrama de influências. 
Ao final, os estudantes utilizaram os recursos digitais para animar o modelo criado, comparando o desempenho do modelo computacional com comportamento descrito pelo diagrama construído em lápis e papel, como pode ser visto na figura 4.

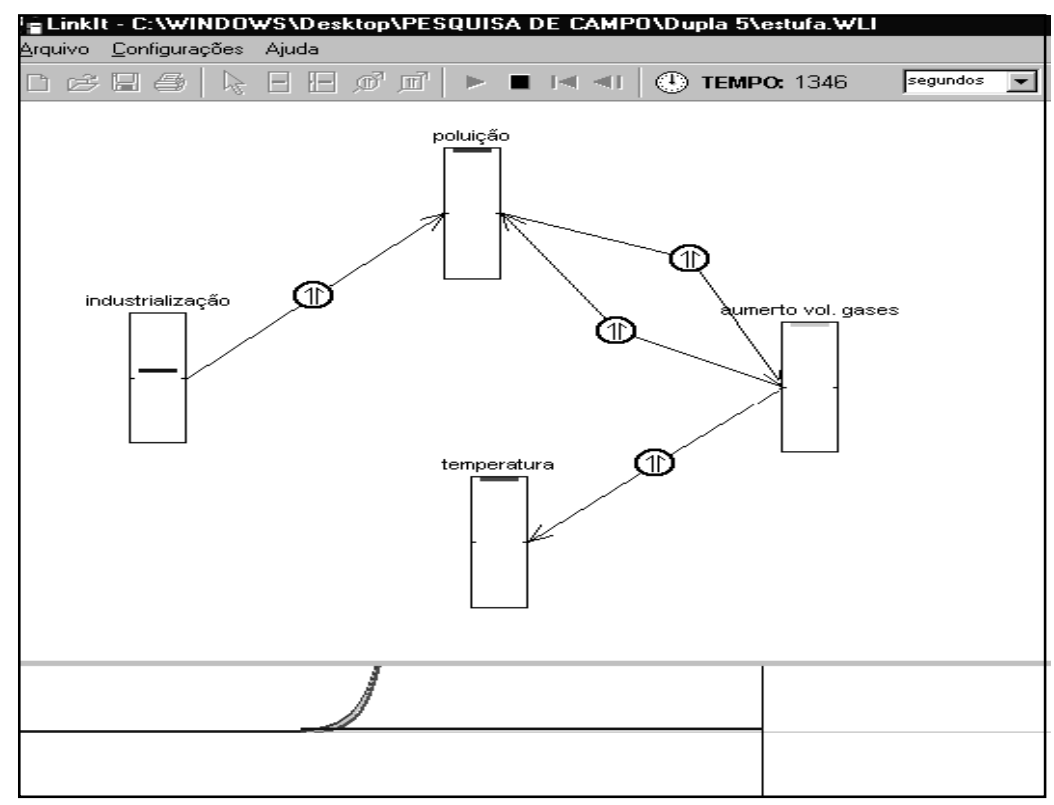

Figura 4 - Modelo computacional do sistema efeito estufa, elaborado pela dupla 05.

No que tange a coleta de dados, utilizou-se os métodos de observação e de análise documental. A observação foi escolhida por ser considerada um método consagrado na obtenção de elementos informativos em uma pesquisa educacional. Ela promove a aproximação entre o observador e o observado, permite a "experiência direta", quando da ocorrência de um determinado fenômeno, e conseqüentemente, aproxima $\mathrm{o}$ observador da visão de mundo do sujeito da pesquisa (Lüdke e André, 1986).

Para realizar as observações trabalhou-se com uma dupla de cada vez e os registros foram efetuados mediante a gravação em vídeo (VHS) do comportamento dos estudantes e do processo de modelagem feito no material instrucional. Ademais, os registros foram incrementados com a gravação digital dos diálogos e a captura digital da tela do monitor do computador, utilizando o software CamStudio (http://www.swftools.com/tool-details.php).

Por sua vez, a análise documental foi escolhida por constituir-se em uma técnica de abordagem de dados qualitativos capaz de complementar as informações advindas de outras técnicas. Ademais, os documentos gerados pelo material textual advindo das atividades exploratórias e expressivas das duplas, possuem as características de estabilidade e riqueza de informações, podendo ser consultados constantemente e servir de base para estudos posteriores (Guba e Lincoln, 1981).

A metodologia escolhida para a categorização, descrição e interpretação dos dados advindos da observação e da análise documental foi a análise de conteúdo, a qual foi realizada através da técnica das redes sistêmicas ${ }^{9}$ de Bliss (1983), com base nos trabalhos de Camiletti (2001), Kurtz dos Santos e Ogborn (1994).

\footnotetext{
${ }^{9}$ Técnica de análise qualitativa cuja proposta é obter uma rede de categorias para descrição de dados. Os elementos básicos para a construção de uma rede sistêmica são o colchete (representa uma escolha exclusiva) e a chave (representa uma escolha simultânea). A leitura da rede dá-se da esquerda para a direita, partindo dos aspectos gerais em direção a um maior detalhamento das categorias.
} 
Dessa forma, construiram-se algumas redes sistêmicas básicas, a partir do exame dos registros contidos nas anotações do pesquisador, bem como, nas gravações de áudio, vídeo e no material escrito das atividades de modelagem, na busca de constituir um grupo de categorias capaz de descrever cada atividade. Na sequência, estas redes foram comparadas umas às outras para dar suporte a possíveis conclusões sobre os processos de construção e validação dos modelos, bem como sobre a qualidade da relação inter-pessoal entre a dupla, objetivando auxiliar na visualização de indícios de habilidades desenvolvidas e de dificuldades encontradas ao longo das atividades.

\section{RESULTADOS E DISCUSSÃO}

A análise das atividades das duplas, com relação às estratégias de modelagem e a interação entre alunos-computador-professor, apresentaram os seguintes resultados. Com relação à conceituação das variáveis ${ }^{10}$ e ligações ${ }^{11}$ para a construção de modelos semiquantitativos dos sistemas físicos panela de pressão e efeito estufa, a maioria das variáveis foram conceituadas como quantidade pelas duplas. Relativo a tal constatação, Kurtz dos Santos e Ogborn (1994) afirmam que conceituar variáveis na forma de quantidades é fundamental para a construção de modelos em nível semiquantitativo. Tais autores apresentam ainda, a possibilidade dos estudantes conceituarem as variáveis como processos $^{12}$ ou eventos ${ }^{13}$, dependendo do sistema em estudo.

Nessa linha de pensamento, Camiletti (2001) comenta a dependência da conceituação das variáveis segundo o tipo de sistema, pois, por exemplo, ao trabalhar com um sistema físico (sistema massa-mola), as variáveis são conceituadas como quantidades e com um sistema biológico (predador-presa) as variáveis são conceituadas como objeto. Em relação a isso, Kurtz dos Santos e Ogborn (1994) comentam que a articulação das variáveis idealizadas como quantidades, durante os diálogos sobre a construção de um modelo, pode indicar a capacidade do estudante em imaginar o mundo em termos de variáveis quantificáveis. No conjunto das duas atividades expressivas, o fato da maioria das ligações terem sido consideradas como causais sugere o uso do raciocínio causal pelos estudantes, o que, segundo Roberts (1983), é importante para a organização de ideias no estudo de sistemas que evoluem no tempo (sistemas dinâmicos). Portanto, quando os alunos qualificaram as variáveis como quantidades e produziram ligações causais entre elas, parece que estavam tentando entender e representar um sistema através do raciocínio semiquantitativo.

Concernente aos procedimentos dos estudantes para elaborar, analisar e validar os modelos envolvidos nas atividades de modelagem exploratória e expressiva, os modelos dos sistemas panela de pressão e efeito estufa foram construídos, primeiro na forma de diagramas de influência e depois na metáfora do software WlinkIt. A estratégia de construção acabou sendo influenciada pelo material instrucional, pois, como os alunos não haviam trabalhado anteriormente com modelagem semiquantitativa, foi considerada a necessidade do material oferecer alguns passos básicos para nortear a aprendizagem dessa técnica. Tais passos foram respectivamente: leitura do texto, definição do sistema

\footnotetext{
${ }^{10}$ A variável é conceituada como quantidade quando seu nome traduz um atributo quantificável, por exemplo: "Nível de pressão interna" ou "Temperatura da Terra". Por outro lado, a variável é considerada como objeto quando seu nome é ligado ao próprio objeto, por exemplo: "água" ou "Terra".

${ }^{11}$ No que tange às ligações entre as variáveis, elas são consideradas causais quando uma variável é alterada pela ação de outra, formando uma relação de influência coerente com as variáveis empregadas e com o sistema estudado (Camiletti, 2001).

12 Processo é uma ação do tipo: "estar comendo", "queima de combustível" ou "reprodução".

${ }^{13}$ Evento é uma ação que pode ser localizada no tempo, exemplo: "iniciar" ou "parar de comer".
} 
a ser estudado, escolha do fenômeno de interesse, escolha das variáveis importantes, construção e descrição do modelo.

Quanto às metáforas (diagrama de influências - figura 2 e computacional - figura 4) da construção inicial dos modelos, como elas são independentes entre si, os estudantes mais experientes puderam optar por uma ou outra. Porém, como o curso oferecido foi introdutório, considerou-se importante a aprendizagem desses dois métodos de representação de modelos, objetivando tornar os alunos aptos a modelar tanto no papel quanto no computador. Quanto à estratégia utilizada para avaliar os modelos, os estudantes realizaram, inicialmente, uma análise teórica, utilizando a técnica de colocar setas acima das variáveis (ver figura 2) para representar um aumento ou diminuição no nível das mesmas, servindo-se dos sentidos de tais setas para descrever o comportamento do modelo e determinar a existência de elo de retroalimentação positivo ou negativo.

Em relação à análise computacional dos modelos, todas as duplas utilizaram a animação de variáveis como auxílio à compreensão do comportamento dos modelos. $\mathrm{O}$ comportamento dos níveis das variáveis foi observado pelos alunos e serviu de base para a discussão sobre a validade dos modelos. Com respeito às saídas gráficas, a maioria das duplas utilizou-as como ponto de referência para detectar problemas e/ou referendar os comportamentos dos modelos. Tal fato pode ratificar os relatos de Camiletti (2001), nos quais as saídas gráficas apresentam-se como um recurso de maior relevância, para a validação do comportamento do modelo, do que a simples animação das variáveis.

Relativamente à validação, esta ocorreu pela comparação entre algumas características do diagrama de influências, do modelo computacional e da concepção sobre o sistema em estudo (formado pelo conhecimento prévio e pelas informações do texto de apoio). Dentre as características usadas para a validação pode-se citar: o comportamento das variáveis (comportamento computacional versus descrição do comportamento do diagrama de influências) e a comparação entre o número de variáveis existentes no diagrama de influências e no modelo computacional. A atividade com o sistema efeito estufa destacou-se pelo alto índice de dificuldades para executar a validação do modelo, sugerindo-se a possibilidade desse fato estar associado à complexidade do sistema, juntamente com a inexperiência dos estudantes em modelagem.

Com relação às habilidades apresentadas pelas duplas durante as atividades de modelagem, foi possível identificar algumas habilidades relativas à construção de modelos semiquantitativos, pois, durante as atividades expressivas, os alunos escolheram as variáveis representativas do sistema, conceberam-nas como quantidades e as interligaram. Dessa forma, estavam determinando uma borda para o sistema, através da qual representaram uma dinâmica interna similar ao sistema em estudo. Com isso, os estudantes exercitaram a criticidade ao escolherem quais variáveis incluir ou não no modelo, conseqüentemente, isso pode ser indício de uma das habilidades do Pensamento Sistêmico denominada "Sistema como causa". Por outro lado, o exame das habilidades apresentadas pelas duplas durante as atividades, tanto expressivas quanto exploratórias indica que a maioria das duplas teve aptidão em discernir os ícones correspondentes a variáveis e ligações, no software WlinkIt.

Além da facilidade no manuseio do software, as duplas apresentaram destreza em transferir corretamente, o modelo em forma de diagrama de influências para a metáfora computacional, ou seja, estavam transpondo o modelo criado para a topologia do software utilizado e, portanto exercendo a habilidade do Pensamento Sistêmico denominada de "Pensamento Operacional". A análise das habilidades desenvolvidas 
apontou para a facilidade em reconhecer a existência e o tipo de elo de retroalimentação dos modelos. Ademais, nas atividades expressivas, a maioria das duplas desenvolveu modelos que utilizaram elos de retroalimentação. A partir dessa constatação é possível inferir o reconhecimento do caráter dinâmico dos sistemas estudados e a tentativa de representar tal dinâmica na estrutura dos respectivos modelos. Como conseqüência, tal comportamento pode indicar o desenvolvimento da habilidade denominada "Pensamento com Elos fechados". Em suma, com base na análise das habilidades e no exame dos modelos construídos, os quais, em sua maioria, apresentaram a característica de conter variáveis com elos causais razoáveis em modelos totalmente coerentes com, pelos menos, um elo de retroalimentação, sugere-se que os estudantes envolvidos na pesquisa, apresentaram indícios da capacidade de desenvolver o Pensamento Sistêmico (Kurtz dos Santos,1995).

Relativo às dificuldades apresentadas pelos estudantes durante as atividades de modelagem notou-se que as duplas foram apresentando um grau crescente de problemas, os quais foram poucos para as atividades exploratórias (talvez porque o modelo já estivesse pronto) e de maior intensidade para as atividades expressivas. Isso parece ter ocorrido porque, nas tarefas expressivas, a demanda intelectual foi de maior monta, pois houve a necessidade de criar um modelo. Nessas atividades, as dificuldades no trabalho com o sistema panela de pressão foram em menor número do que na tarefa com o sistema efeito estufa, quanto a essa constatação, é recomendável levar em conta o fato do sistema panela de pressão ser mais simples e ter maior proximidade com o cotidiano do estudante.

Para deliberar sobre o processo de interação dos componentes das duplas entre si e com o professor, foram utilizadas algumas categorias como: participação positiva da dupla (ação solícita e construtiva) quando solicitada pelo professor; interações espontâneas e construtivas entre os componentes das duplas (compartilhamento de informações e auxílio mútuo ao longo das atividades); capacidade de expressar-se com clareza; respeito à escala de valores do outro; comprometimento com acordos realizados; percepção de fatores perturbadores; descrição e solução de situaçõesproblema; atitudes de curiosidade e criatividade (Xavier, 2000).

A análise das atividades, segundo as categorias supracitadas, sugere a existência de atitudes de reciprocidade entre os componentes das duplas, denotando a ocorrência de respeito mútuo e a predisposição para a participação em trocas colaborativas. Portanto, é plausível supor a existência de atitudes de cooperação durante as tarefas de modelagem. Nesse sentido, busca-se o apoio de Piaget (1977), o qual coloca a autonomia como paralela à cooperação, caracterizando-a pela liberdade de decisão, criatividade e curiosidade e de Ramos (1996), que a vincula à capacidade de percepção de problemas impeditivos ao sucesso em uma determinada ação, para propor a possibilidade dos estudantes, em alguns momentos, terem assumido o controle da sua aprendizagem configurando assim atitudes de autonomia. Em suma, o exame global das categorias envolvidas no processo de interação das duplas recomenda a possibilidade das atividades de modelagem computacional terem auxiliado os estudantes na ampliação da capacidade de perceber a si mesmos e a sociedade onde estão inseridos. Portanto, tais atividades parecem ser uma maneira do aluno construir e reconstruir seu conhecimento, desenvolvendo algumas habilidades que permitirão uma maior qualidade de vida e que são importantes para o mercado de trabalho atual, como: cooperação, criatividade, capacidade de aprender e autonomia.

Com respeito às possibilidades e limitações do software WlinkIt para a execução das atividades computacionais, o primeiro aspecto a ser mencionado foi a rápida e fácil adaptação dos alunos quanto à metáfora apresentada e quanto ao manuseio dos controles 
de animação e de configuração das variáveis. Os procedimentos para construção e animação dos modelos foram rapidamente apreendidos pelas duplas. Quanto às saídas gráficas, as mesmas ainda permitem somente gráficos das variáveis em relação ao tempo e não umas em relação às outras, as duplas não sentiram falta desse recurso, mas sua implementação poderia ser útil para aumentar as informações sobre os modelos. Outra sugestão seria aumentar o tamanho das telas gráficas e torná-las independentes da área de trabalho do software. Sob o aspecto geral, a contribuição desse ambiente computacional foi positiva, mostrando-se um instrumento valioso no trabalho com modelagem computacional semiquantitativa.

\section{CONCLUSÕES}

Este trabalho foi importante por ser inédito na aplicação da modelagem computacional semiquantitativa, utilizando o software WlinkIt, com estudantes do Ensino Médio de uma escola pública. Ademais, acredita-se que foram revelados indicativos a respeito da capacidade dos estudantes em trabalhar com modelagem e sobre a possibilidade do desenvolvimento do raciocínio semiquantitativo. Ao longo dos trabalhos os estudantes puderam praticar a leitura, a escrita e a reflexão sobre seus julgamentos. Tal procedimento é interessante para os alunos do Ensino Médio, especialmente do turno da noite pois, em geral, os mesmos apresentam carências quanto à interpretação e desenvolvimento de textos. Outro aspecto relevante desta investigação foi a evolução técnica em relação às pesquisas antecedentes envolvendo a modelagem computacional, pois, anteriormente, a coleta de dados era feita pela gravação (em VHS) da tela do monitor e das vozes dos alunos. Porém, neste trabalho, procurou-se utilizar, ao máximo, os recursos de um computador pessoal, tanto para coletar quanto para armazenar os dados.

Dessa forma, as atividades computacionais (som e imagem) foram registradas, digitalmente, via software CamStudio, na forma de arquivos com extensão AVI. Com isso, a qualidade das imagens melhorou muito, sem contar a facilidade de armazenamento das mesmas, facilitando o transporte e a apresentação. Também foi utilizada uma Webcam para armazenar o processo de construção dos modelos no papel. Com isso, acredita-se ter coletado uma maior quantidade de dados, enriquecendo o trabalho.

Ademais, o software CamStudio (encontrado, gratuitamente, na página http://www.swftools.com/tool-details.php) poderá ser utilizado para produzir arquivos tutoriais sobre modelagem, os quais, caso sejam disponibilizados na internet, por exemplo, na página do ModelCiências (http://www.modelciencias.furg.br/), da FURG, poderão ser usados na aprendizagem, à distância, de modelagem.

Por fim, espera-se ter contribuído para o debate sobre o ensino e a aprendizagem de Física no Nível Médio e sobre a aplicação educacional da modelagem computacional semiquantitativa.

\section{REFERÊNCIAS BIBLIOGRÁFICAS}

BLISS, J. Qualitative Data Analysis for Education Research: a guide of systemic network. London: Croom Helm, 1983. 
CAMILETTI, G. G. A modelagem computacional semiquantitativa no estudo de tópicos de ciências: um estudo exploratório com estudantes universitários. Vitória: UFES, 2001. 218p. Dissertação de Mestrado.

GUBA, E.G. e LINCOLN, Y. S. Effective Evaluation. San Francisco: Jossey Bass, 1981.

KURTZ DOS SANTOS, A.C. OGBORN, J. Sixth form students' ability to engage in computational modeling. Journal of Computer Assisted Learning. Vol 10, p. 182-200, 1994.

KURTZ DOS SANTOS, A.C.K. Introdução à modelagem computacional na educação. Rio Grande: FURG, 1995.

LÜDKE, M. e ANDRÉ, M. Pesquisa em Educação: abordagens qualitativas. São Paulo: EPU, 1986.

MACHADO, B. S.; KRÜGER, K. Utilizando o STELLA no Laboratório de Física. Anais do III Seminário sobre Representações e Modelagem no Processo de EnsinoAprendizagem. Rio Grande: Fundação Universidade do Rio Grande. 17-19/Novembro 1999. p. 44-49.

MANDINACH, E. B. Model-building and the Use of Computer Simulation of Dynamics Systems. J. Educational Computing Research. 1989.

MORIN, E. Introdução ao pensamento complexo. Lisboa: Instituto Piaget, 1991.

OGBORN, J. Modelling whit the Computer at all ages. Publicação interna do Institute of Education University of London, 1992.

PIAGET, J. O julgamento moral na criança. São Paulo: Editora Forense, 1977.

RAMOS, E. Análise ergonômica do sistema hiper Net buscando o aprendizado da cooperação e da autonomia. Florianópolis: PPEP/UFSC, 1996. Tese de doutorado.

ROBERTS, N. Introduction to Computer Simulation - a System Dynamics Modelling Approach. New York: Addison Wesley, 1983.

SAMPAIO, F.F. Modelagem Dinâmica Computacional e o Processo de EnsinoAprendizagem: algumas questões para reflexão, Rio Grande, 1999. Disponível em: < http://www.cienciaemtela.nutes.ufrj.br/artigos/0109sampaio.pdf > Acesso em: $17 \mathrm{de}$ julho de 2015.

XAVIER, R. T. O. O construcionismo e o desenvolvimento da cooperação, da autonomia e da auto-estima. Pelotas: PPGE/UFPEL, 2000. 171p. Dissertação de Mestrado. 